\title{
Use of PocketMaker Microkeratome for Creation of Corneal Pocket for Foldable Keratoprosthesis KeraKlear Implantation - Case Series
}

\author{
Pavel Studeny, Deli Krizova* and Pavel Kuchynka \\ Department of Ophthalmology of $3^{\text {rd }}$ Medical Faculty and University Hospital Kralovske Vinohrady, Prague, Czech \\ Republic
}

\begin{abstract}
Purpose: To describe a surgical method for corneal pocket creation for KeraKlear keratoprosthesis implantation by PocketMaker microkeratome.

Methods: We implanted keratoprosthesis KeraKlear in 3 patients. In all cases, we used a microkeratome PocketMaker to create a corneal pocket, where the incision was made at a depth of $300 \mu \mathrm{m}$ with a vibrating diamond blade.

Results: Although corneas have been extensively opaque and vascularized, in all three cases we successfully performed suction of the microkeratome system and created a corneal pocket without any difficulties. Subsequent keratoprosthesis implantations were performed without any problems.
\end{abstract}

Conclusion: The technique is simple, relatively cheap, and the creation of the corneal pocket is possible even in patients with vascularized and opaque cornea.

Keywords: Corneal pocket, keratoprosthesis KeraKlear, PocketMaker.

\section{INTRODUCTION}

The KeraKlear (Keramed, USA) artificial cornea is a medical device employed in the treatment of corneal blindness. It is a non-perforating foldable corneal prosthesis which is implanted into a partial thickness corneal pocket made with a femtosecond laser through an opening of 3.5 $\mathrm{mm}$ in diameter. The recommended depth of the pocket is $300 \mu \mathrm{m}$. The deeper part of corneal stroma, the Descemet's membrane and endothelium are left in place. The superior part of the cornea is cut in the central $3.5 \mathrm{~mm}$ region and removed. The optical part of keratoprosthesis is placed in this opening. The main function of the peripheral part is to fixate and stabilize the keratoprosthesis in the correct position. It is inserted between the superior and the deep lamellae of the corneal pocket. The femtosecond laser is recommended for corneal pocket creation. This technique has some limitations. The main disadvantages are the difficulties experienced in vacuum creation between the cornea and the suction ring of the femtosecond laser, especially in cases of corneal and conjunctival scaring or an irregular corneal surface. Laser discision of the tissue in completely white cornea may be a further problem. Unfortunately, irregular surfaces as well as a white cornea are very frequent in patients indicated for keratoprosthesis implantation.

An alternative technique for creating a corneal pocket involves the PocketMaker microkeratome (Dioptex GmbH,

*Address correspondence to this author at the Department of Ophthalmology of $3^{\text {rd }}$ Medical Faculty and University Hospital Kralovske Vinohrady, Srobarova 50, 10034 Prague, Czech Republic;

Tel: +420267162835; E-mail: deli.krizova@fnkv.cz
Austria). This equipment was originally used in the creation of a corneal pocket and the implantation of a MyoRing (Dioptex GmbH, Austria). MyoRing is an intracorneal continuous ring (ICCR) and was designed by Daxer for the treatment of myopia [1] and keratokonus [2,3]. The discision of a cornea and the creation of a pocket in this technique is performed by using a vibrating diamond blade after the previous applanation of the cornea using a plastic applanator. The depth of the corneal pocket created by a PocketMaker microkeratome is $300 \mu \mathrm{m}$, so we tried to use this method to create a corneal pocket for our keratoprosthesis KeraKlear implantation.

\section{MATERIALS AND METHODS - SURGICAL TECHNIQUE}

The surgical procedure starts with the creation of a pocket in the corneal stroma using the PocketMaker microkeratome. It consists of a suction ring; an applicator with a guiding mean for the handpiece; a disposable transparent applanator; a handpiece containing a motordriven diamond blade that vibrates in the cutting plane; and a control unit. The first step of the procedure is to fixate the applicator to the eye using the suction ring. The cornea is applanated using an applanator, inserted into the applicator (Fig. 1). After inserting the handpiece into the guiding mean, the cornea is dissected into 2 lamellae with a vibrating diamond blade. The depth of the corneal pocket is $300 \mu \mathrm{m}$ and the diameter is approximately $9.0 \mathrm{~mm}$. Apart from a 3-4 $\mathrm{mm}$ wide incision tunnel, the pocket is closed along the entire circumference. The suction ring is removed from the eye. A plastic glide is inserted into the pocket via the incision tunnel. In the central part, the superior $300 \mu \mathrm{m}$ of the cornea is cut over the glide, using a $3.5 \mathrm{~mm}$ disposable corneal punch (Biopsy punch, Kai Medical, Japan). The part 


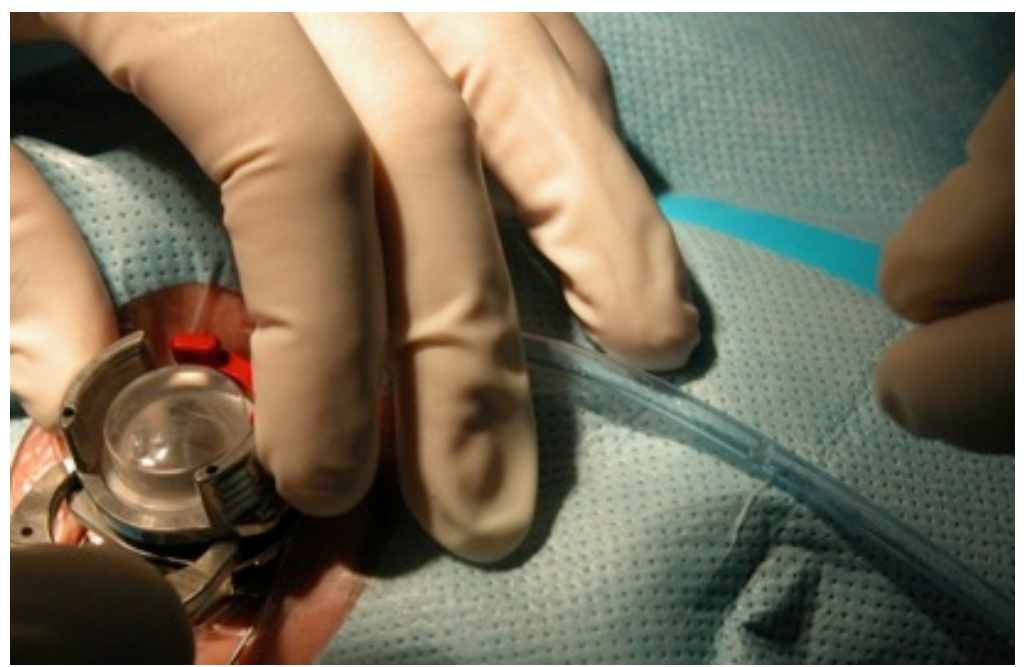

Fig. (1). Fixation of the applicator to the eye using a suction ring and applanation of the cornea using an applanator.

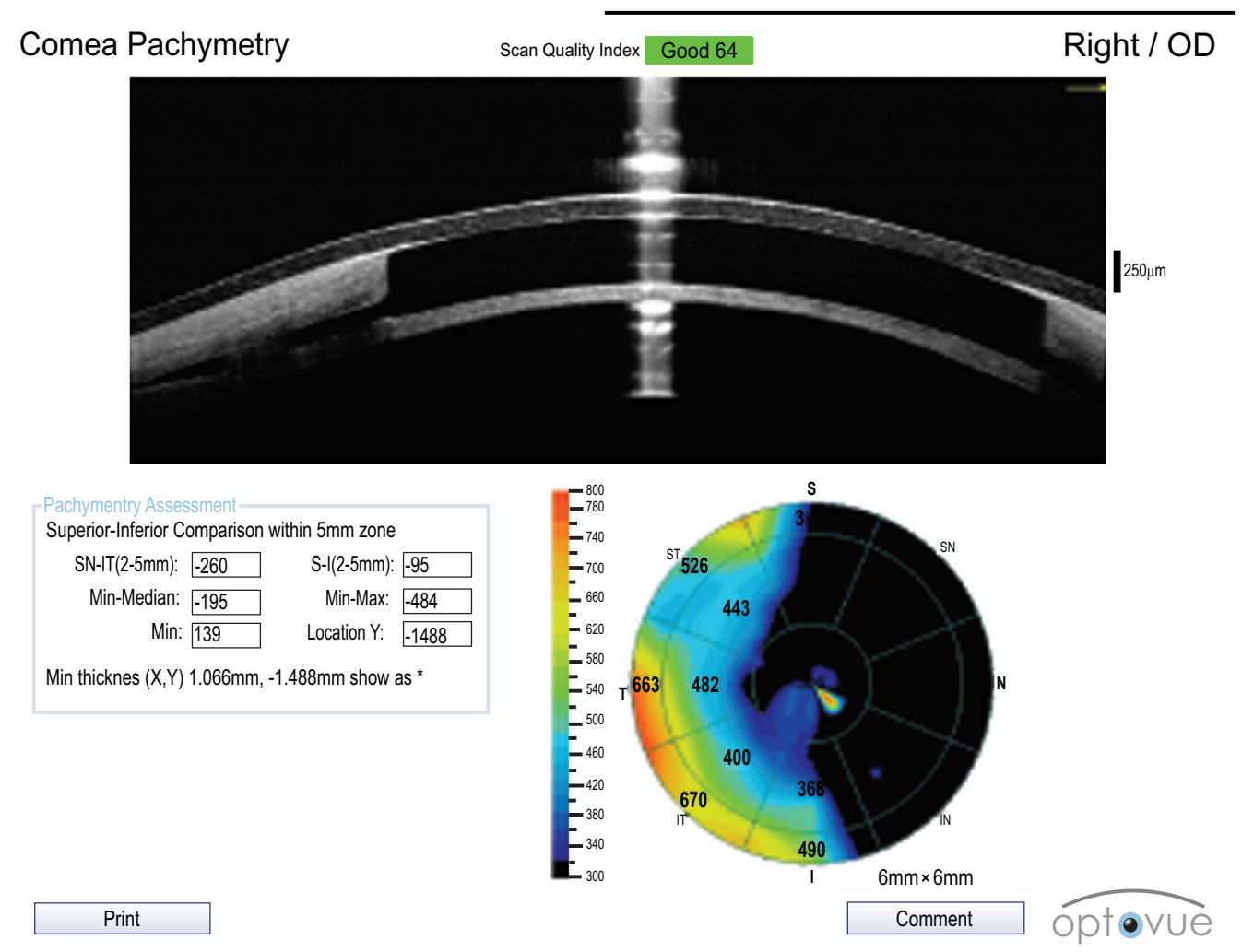

Fig. (2). Anterior segment OCT scan of the implanted KeraKlear.

of the cornea cut from the center is removed, as is the glide. KeraKlear keratoprosthesis is made of a soft and foldable material so it can be implanted using forceps into the corneal pocket through the $4 \mathrm{~mm}$ incision. The forceps are released after the implantation into the pocket and the prosthesis unfolds spontaneously. The central optical part fills the 3.5 $\mathrm{mm}$ hole in the center of the cornea and the thinner peripheral part of the prosthesis is inserted between the superficial and deep lamellae of the corneal pocket in the periphery. The position of the keratoprosthesis can be controlled or corrected using a cyclospatula. The main function of the peripheral rim is to fixate and stabilize the keratoprosthesis in the correct position (Fig. 2). This part of the prosthesis contains 12 holes and allows for fixation with a suture. We always used 4 single sutures, one in each quadrant. The incision tunnel is self-sealing and does not require suturing. A contact lens is applied at the end of the surgery. The whole surgery is fast and relatively easy. It is possible to perform this surgery under topical anesthesia. 
(a)

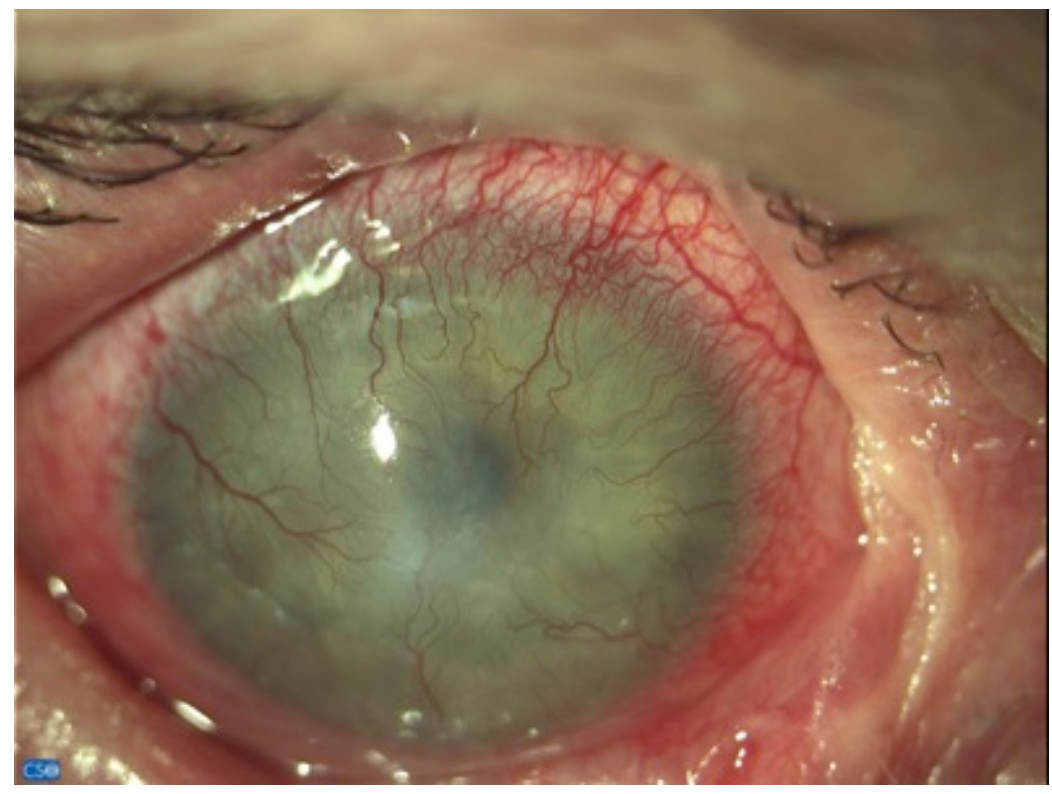

(b)

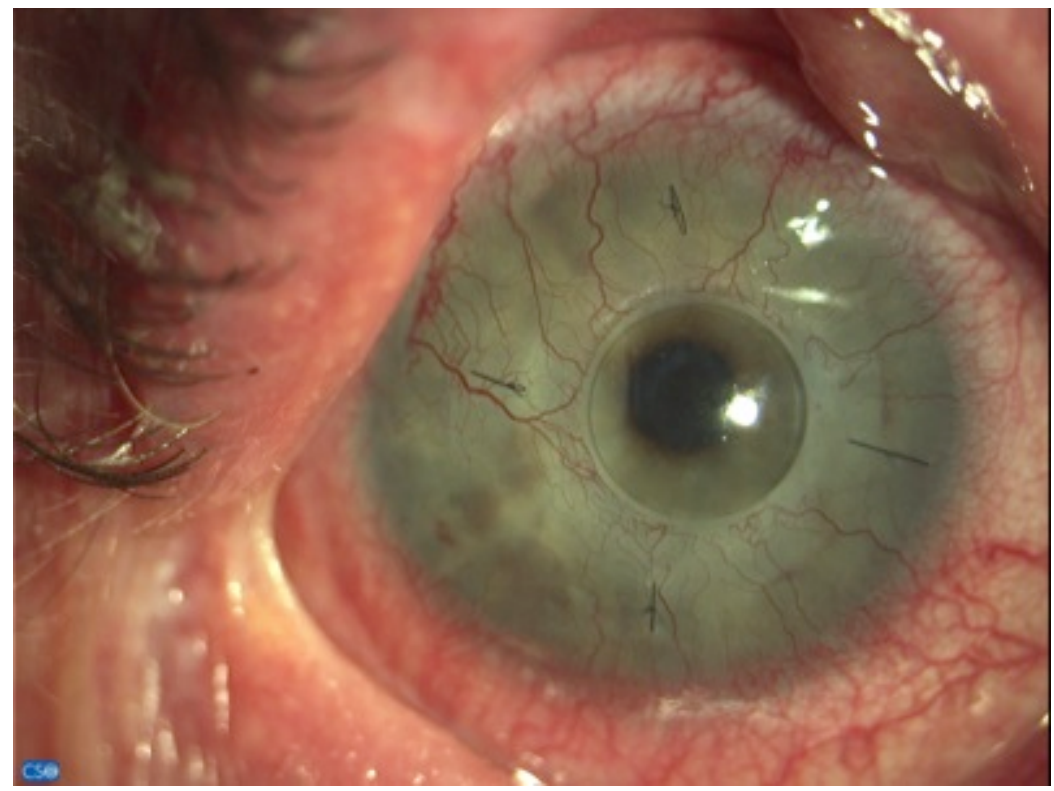

Fig. (3). Patient 1. (a) Cornea before surgery and (b) 6 months after surgery.

The KeraKlear keratoprosthesis and PocketMaker microkeratome are both $\mathrm{CE}$ certified and are approved for use in surgery in the European Union.

\section{RESULTS}

\section{Patient 1}

62 years old female, suffered from an attack of Lyell's syndrome 30 years ago. She underwent penetrating keratoplasty 5 times in her left eye. The last surgery was performed 6 years ago. There was no keratoplasty performed in the right eye. The visual acuity of the right eye was initially slightly better, but gradually also deteriorated. The best corrected distance visual acuity (CDVA) measured on Snellen optotypes preoperatively was 0.01 decimal in the right eye and 0.05 in the left eye. In June 2011 we implanted the KeraKlear keratoprosthesis into the corneal pocket created with the PocketMaker microkeratome. The implant was fixed with 4 single stitches (Fig. 3a, b). The surgery was performed under general anesthesia. One week after surgery, the CDVA was 0.2 . At 6,12 and 24 months after surgery, the CDVA was 0.4 . The patient is wearing myopic contact lens $(-12 \mathrm{sphD})$ and she is permanently using topical antibiotics.

\section{Patient 2}

82 years old female, underwent two penetrating keratoplasties for corneal leucoma after herpetic keratitis in her left eye. The right eye was severely amblyopic with no other ocular disease. The CDVA before surgery was hand motion in both eyes. The surgery was performed under 
topical anesthesia. The surgical procedure was the same as that described in case 1. On the first postoperative day, the keratoprosthesis was slightly loose. There was a tiny gap between the edge of the cornea and the optical part of the keratoprosthesis. We resutured the keratoprosthesis with continuous suture. All other postoperative care was the same as that in the previous case. The visual acuity was 0.1 week after surgery. At 6,12 and 18 months after surgery, the visual acuity was $0.1,0.25$ and 0.1 respectively. The reason for the minor decrease in visual functions in the 18th month is probably a fine fibrosis of the posterior corneal lamellae under the keratoprosthesis.

\section{Patient 3}

77 years old male, suffered alkali burns to both eyes 40 years ago. In the past, he underwent repeated penetrating keratoplasty and cataract surgery in both eyes. Keratoplasty was performed twice on the right eye and 3 times on the left eye. The CDVA before surgery was hand motion in both eyes. We implanted the keratoprosthesis KeraKlear into the patient's right eye (Fig. 4a, b). The surgical procedure was the same as that in the previous cases. Surgery was performed under topical anesthesia. 1 week, 1 month and 6 months after surgery, the CDVA was 0.1 ; the patient wore myopic contact lens ( $-13 \mathrm{sphD})$ after surgery. Unfortunately the patient died 8 months after surgery.

\section{DISCUSSION}

Currently, the most widely used keratoprostheses are Boston keratoprosthesis, osteoodontokeratoprosthesis and Alphacor. They are used primarily in patients with bilateral corneal blindness and in cases of high risk for penetrating

(a)

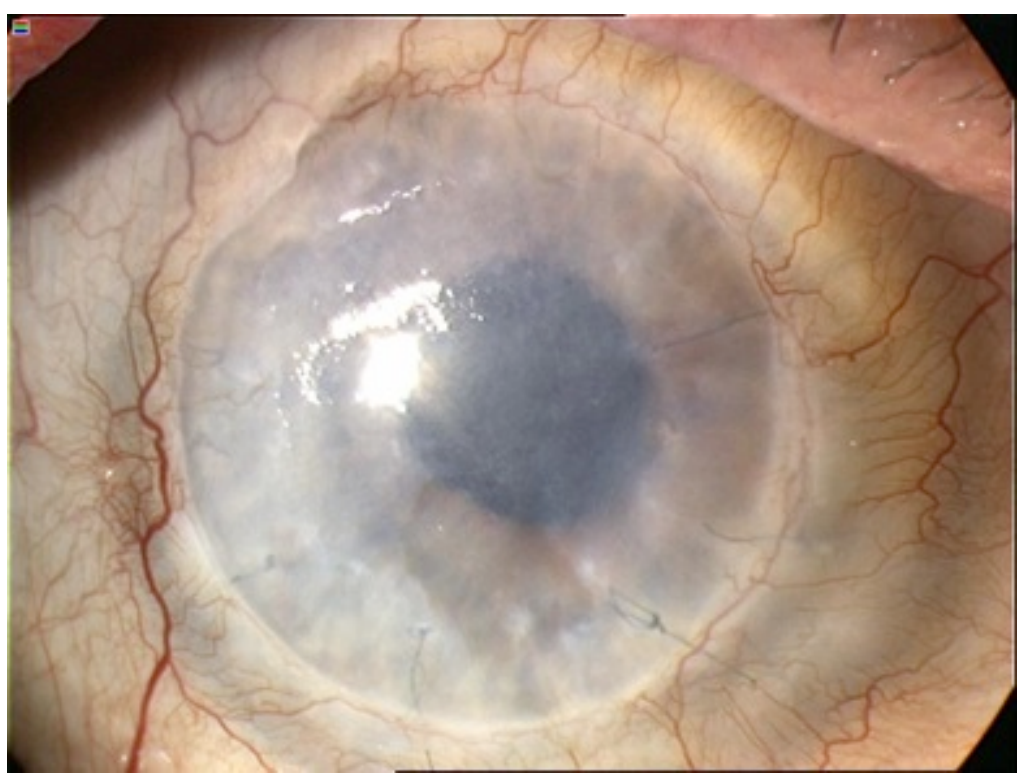

(b)

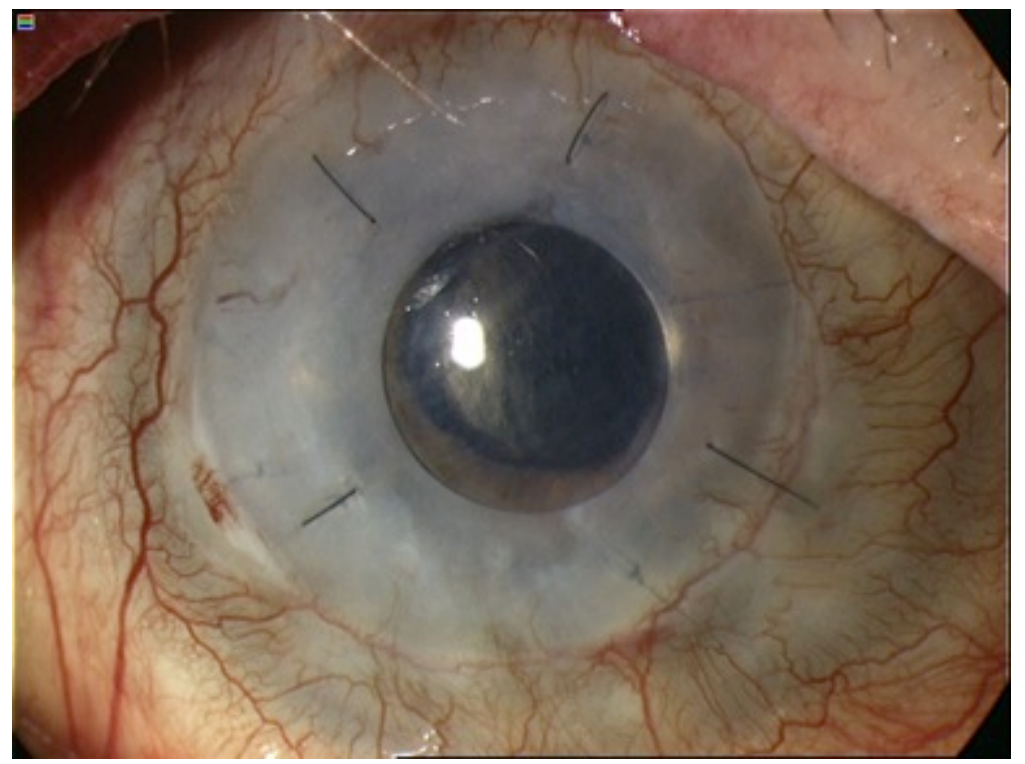

Fig. (4). Patient 3. (a) Cornea before surgery and (b) 6 months after surgery. 
keratoplasties. Despite the relatively promising results in some patients, keratoprostheses are associated with many risks, including peroperative and postoperative complications. The main postoperative complications in patients with implanted keratoprosthesis are glaucoma, a retroprosthetic membrane, chronic hypotony, dehiscence between the artificial material and the recipient's corneal tissue, and the consequent risk of endophthalmitis [4-9]. These complications often cause deterioration of visual functions and failure of the surgery. In comparison with standard keratoprostheses, KeraKlear has a great advantage, since the surgery is non-penetrating. The artificial cornea is placed on a thin layer of the recipient's posterior cornea. The risk of severe complications is theoretically greatly reduced. Since the retained layer of the cornea is thin, it allows a patient with implanted artificial cornea a relatively good visual acuity. The prosthesis is fixed in the correct position using a peripheral rim implanted into the corneal pocket. In our experience, when fixing the rim, 4 single sutures are sufficient to ensure the long-term stability of the implant.

An important step in the prosthesis implantation procedure is to create a corneal pocket. The recommended use of the femtosecond laser has some disadvantages, especially when creating the vacuum in cases of a scarred and irregular corneal surface, as it is problematic to cut on a clouded and vascularized cornea. One particular disadvantage is the high cost of the device (femtosecond laser) and the relatively expensive procedure. When using the PocketMaker, the corneal pocket is created by a vibrating diamond knife. Suction of the microkeratome head is possible even in relatively opaque cornea. Corneal opacification or vascularization does not present any complication when creating a corneal pocket. All parts of the PocketMaker are reusable, therefore the device is relatively cheap. In addition, all parts of the PockerMaker, including the control panel, can be put into a small suitcase and easily transported. This makes it possible to perform the surgery at a clinic where no femtosecond laser is available. Regarding the surgical procedure, it is very simple and fast, and it can be performed under topical anesthesia.

\section{CONCLUSION}

The artificial cornea KeraKlear is potentially a very useful medical device in the treatment of corneal blindness.
Unlike other types of keratoprostheses, the implantation of a KeraKlear is a non-perforating surgery. This theoretically lowers the risk of serious intraoperative and postoperative complications, such as endophthalmitis, bleeding, damage of intraocular tissue and secondary glaucoma. According to our experience, the PocketMaker, a vibrating diamond knife used to create a corneal pocket for the implantation of a keratoprosthesis, is an advisable, simple and cheap alternative to the recommended use of the femtosecond laser.

\section{CONFLICT OF INTEREST}

The authors confirm that this article content has no conflict of interest.

\section{ACKNOWLEDGEMENTS}

Declared none.

\section{REFERENCES}

[1] Daxer A. Corneal intrastromal implantation surgery for the treatment of moderate and high myopia. J Cataract Refract Surg 2008; 34: 194-8.

[2] Daxer A, Mahmoud H, Venkateswaran RS. Intracorneal continuous ring implantation for keratoconus: One-year follow-up. J Cataract Refract Surg 2010; 36: 1296-302.

[3] Mahmoud H, Venkateswaran RS, Daxer A. Implantation of complete corneal ring in an intrastromal pocket for keratoconus. J Refract Surg 2011; 27: 63-8.

[4] Munoz-Gutierrez G, Alvarez de Toledo J, Barraquer RI, et al. Postsurgical visual outcome and complications in Boston type 1 keratoprosthesis. Arch Soc Esp Oftalmol 2013; 88: 56-63.

[5] Dokey A, Ramulu PY, Utine CA, et al. Chronic hypotony associated with the Boston typ 1 keratoprosthesis. Am J Opththalmol 2012; 154: 266-71.

[6] Aldave AJ, Snagwan VS, Basu S, et al. International results with the Boston typ I keratoprosthesis. Ophthalmology 2012; 119: 15308 .

[7] Tan A, Tan DT, Tan XW, Mehta JS. Osteo-odonto keratoprosthesis: systematic rewiew of surgical outcomes and complication rates. Ocul Surf 2012; 10: 15-25.

[8] Cahn CC, Holland EJ. Infectious endophthalmitis after Boston typ 1 keratoprosthesis implantation. Cornea 2012; 31: 346-9.

[9] Hicks CR, Crawford GJ, Lou X, et al. Corneal replacement using a synthetic hydrogel cornea, alphacor: device, preliminary outcomes and complications. Eye (Lond) 2003; 17: 385-92. 